\title{
3
}

\section{COMPORTAMIENTO DEL CULTIVO DEL LIMON (Citrus aurantifolia Swingle) EN DOS LOCALIDADES DE SANTA ELENA, ECUADOR.}

Mercedes Santistevan Méndez, Salomón Helfgott Lerner, Oscar Loli Figueroa, Alberto Julca Otiniano 


\title{
COMPORTAMIENTO DEL CULTIVO DEL LIMON (Citrus aurantifolia Swingle) EN DOS LOCALIDADES DE SANTA ELENA, ECUADOR
}

\author{
Mercedes Santistevan Méndez; Salomón Helfgott Lerner; Oscar Loli Figueroa; Alberto Julca Otiniano \\ Universidad Nacional Agraria La Molina (UNALM). Escuela de Posgrado. Programa de Doctorado \\ Agricultura Sustentable (PDAS). Apto. 12056. La Molina. Lima. Perú.
}

\begin{abstract}
Resumen
Se realizó una investigación entre agosto y diciembre del 2015, con el objetivo de conocer el comportamiento del cultivo de limón (Citrus aurantifolia Swingle) en dos localidades de la Provincia de Santa Elena, Ecuador. Se seleccionaron dos plantaciones de ocho años de edad, en Manglaralto y Colonche. En cada localidad se marcaron 50 plantas, en las que se hicieron las evaluaciones. El ensayo no tuvo un diseño experimental, pero el análisis estadístico se hizo como si fuera un Diseño Completamente al Azar (DCA), considerando cada localidad como un tratamiento y cada planta como una repetición. Se encontró que la mayor incidencia de plagas y enfermedades se presentó en Manglaralto. El mayor diámetro del fruto, correspondió a Colonche, con una diferencia estadísticamente significativa con respecto a Manglaralto. El mayor peso del fruto y el mayor rendimiento, correspondió a Colonche; pero las diferencias no fueron estadísticamente significativas con respecto a Manglaralto.
\end{abstract}

Palabras claves: cítricos - plagas - enfermedades - calidad-fruto

\begin{abstract}
An investigation was conducted between August and December 2015 in order to understand the behavior of the lemon crop (Citrus aurantifolia Swingle) at two locations in the Province of Santa Elena, Ecuador. Two eight years old commercial plantations were selected in Manglaralto and Colonche. In each location, 50 plants were labeled in order to be evaluated. The trial did not have an experimental design but statistical analysis was done like a Completely Randomized Design (CRD), considering each location as a treatment and each plant as a replicated. It was found that the highest insect and disease incidence to plays in Manglaralto. The greatest fruit diameter was found in Colonche, with a statistically significant difference with respect to Manglaralto. The highest fruit weight and yield performance was found in Colonche; but the differences were not statistically significant with respect to Manglaralto.
\end{abstract}

Keywords: citrus - pests - diseases - quality - fruit 


\section{Introducción}

El limón Sutil (Citrus aurantifolia Swingle) es la especie de cítrico más cultivada en el Ecuador, lo sigue el limón Tahití (Citrus latifolia Tan), entre ambos suman aproximadamente unas 4400 ha cultivadas. En la Provincia de Santa Elena, hay 500 ha de limón sutil y las perspectivas de aumentar el área sembrada son alentadoras ya que es uno de los cultivos que viene apoyando el Proyecto Integral de Desarrollo Agrícola Ambiental y Social de forma Sostenible (PIDAASSE). En esta provincia, Manglaralto y Colonche, son las dos localidades de mayor producción; pero no existe información básica documentada sobre el comportamiento de este cultivo en esta zona. Por otra parte en reuniones con técnicos y agricultores de la provincia, se ha sugerido la necesidad de realizar investigación agronómica, para ayudar a la mejora tecnológica de este cultivo en Santa Elena. Por ello, este trabajo de investigación se realizó con el objetivo de conocer el comportamiento del cultivo de limón en dos localidades de la Provincia de Santa Elena, Ecuador.

\section{Materiales y Métodos}

El estudio se realizó entre agosto y diciembre del 2015, para ellos se seleccionaron dos fincas productoras de limón de ocho años de edad, en Manglaralto y Colonche, en cada una de estas, se marcaron 50 plantas, en las que se realizó un seguimiento durante los cinco meses para las evaluaciones respectivas. Previamente se tomó muestras del suelo de cada zona, las mismas que fueron enviadas al Laboratorio de Suelos de la Estación Experimental INIAP Boliche en el Guayas, Ecuador, las variables evaluadas fueron: $\checkmark \quad$ Incidencia de plagas y enfermedades.- La incidencia de las principales Plagas y Enfermedades se evaluó mensualmente durante los 5 meses que duró la investigación, para ello se marcaron 50 plantas.

$\checkmark \quad$ Rendimiento.- Se evaluó a las 50 plantas previamente marcadas se hicieron un total de 20 cosechas ("pasadas"), es decir una vez por semana y los datos se procesaron para cada mes.

$\checkmark \quad$ Calidad del limón.- Para ello, en cada uno de las 20 cosechas ("pasadas"), del total de frutos, se tomó una muestra al azar de 10 frutos, los cuales se tomó el peso y el diámetro usando una balanza analítica y un vernier respectivamente. Llos datos se procesaron para cada mes.

El ensayo no estuvo bajo un diseño experimental; pero el análisis estadístico se hizo como si fuera un Diseño Completamente al Azar (DCA), considerando cada localidad como un tratamiento y cada planta de limón como una repetición.

\section{Resultados y Discusión}

Manglaralto y Colonche son dos zonas ecológicas poseen condiciones climáticas que pertenecen a la misma provincia. Las condiciones climáticas son bastante parecidas, aunque los valores son ligeramente mayores en la primera localidad, tanto para la temperatura como precipitación anual, que son de 26 y $25^{\circ} \mathrm{C}$ y 200 - 300 y 125 - $150 \mathrm{~mm}$, respectivamente INAMHI [6]. Los suelos son de textura franco-arcilloso en la primera localidad y franco en Colonche; los valores de $\mathrm{pH}, \mathrm{N}, \mathrm{P}, \mathrm{K}$ y $\mathrm{Ca}$, son bastante parecidos en ambas localidades; pero el contendido de $\mathrm{Mg}$ es mayor en Colonche (Cuadro 1).

Cuadro 1.Análisis de suelo de las zonas de Colonche y Manglaralto en la provincia de Santa Elena, Ecuador.

\begin{tabular}{|l|c|c|c|c|c|c|}
\hline Zona Agroecológica & $\mathrm{pH}$ & $\begin{array}{c}\mathrm{N} \\
(\mathrm{ppm})\end{array}$ & $\begin{array}{c}\mathrm{P} \\
(\mathrm{ppm})\end{array}$ & $\begin{array}{c}\mathrm{K} \\
(\mathrm{meq} / 100 \mathrm{ml})\end{array}$ & $\begin{array}{c}\mathrm{Ca} \\
(\mathrm{meq} / 100 \mathrm{ml})\end{array}$ & $\begin{array}{c}\mathrm{Mg} \\
(\mathrm{meq} / 100 \mathrm{ml})\end{array}$ \\
\hline Colonche & 7.2 & 23 & 38 & 2.40 & 18.88 & 8.14 \\
\hline Manglaralto & 7.5 & 24 & 33 & 2.32 & 18.02 & 4.75 \\
\hline
\end{tabular}




\section{A.- Incidencia de plagas y enfermedades}

Se reportó la presencia de pulgones, mosca blanca, ácaros, hormigas y fumagina problemas sanitarios muy comunes en limón y otras especies cultivadas de cítricos, tanto en Ecuador [4]; [5] como en otros países productores como en Perú Vegas, y Narrea, [12]. En Colonche, los pulgones solamente se presentaron en diciembre; mientras que en Manglaralto estuvo presente en todo el periodo de estudio, con excepción de los meses de octubre y noviembre. En ambas localidades, la mosca blanca, no fue reportada en los meses de octubre y noviembre; pero sí en agosto, setiembre y diciembre; mientras que los ácaros solamente se presentaron en agosto y setiembre. Las hormigas, solo se encontraron en Manglaralto, en casi todo el periodo de estudio; con excepción de agosto. $\mathrm{La}$ fumagina, solamente se reportó en Manglaralto, en casi todo el periodo de estudio; con excepción de los meses de octubre y noviembre. Los niveles de incidencia de estas plagas en Manglaralto, hizo necesaria la aplicación de medidas de control químico a fines de setiembre, lo que explicaría, la ausencia de estos los problemas en los dos meses siguientes.

Cuadro 2. Incidencia de plagas y enfermedades (\%) en Colonche y Manglaralto, provincia de Santa Elena, Ecuador.

\begin{tabular}{|c|c|c|c|c|c|c|}
\hline \multirow{2}{*}{$\begin{array}{c}\text { Zona } \\
\text { Agroecológica }\end{array}$} & \multirow[b]{2}{*}{ Meses } & \multicolumn{5}{|c|}{ Incidencia mensual (\%) } \\
\hline & & Pulgones & Mosca Blanca & Hormigas & Ácaros & Fumagina \\
\hline \multirow{5}{*}{ Colonche } & Agosto & 0 & 5 & 0 & 4 & 0 \\
\hline & Septiembre & 0 & 8 & 0 & 4 & 0 \\
\hline & Octubre & 0 & 0 & 0 & 0 & 0 \\
\hline & Noviembre & 0 & 0 & 0 & 0 & 0 \\
\hline & Diciembre & 5 & 7 & 0 & 0 & 0 \\
\hline \multirow{5}{*}{ Manglaralto } & Agosto & 15 & 25 & 6 & 2 & 28 \\
\hline & Septiembre & 23 & 25 & 9 & 2 & 33 \\
\hline & Octubre & 0 & 0 & 0 & 0 & 0 \\
\hline & Noviembre & 0 & 0 & 9 & 0 & 0 \\
\hline & Diciembre & 8 & 13 & 8 & 0 & 20 \\
\hline \multicolumn{2}{|l|}{ Promedio Colonche } & $1.0 \mathrm{a}$ & $4.0 \mathrm{a}$ & $0.0 \mathrm{a}$ & $0.8 \mathrm{a}$ & $0.0 \mathrm{a}$ \\
\hline \multicolumn{2}{|l|}{ Promedio Manglaralto } & $9.2 \mathrm{a}$ & $12.6 \mathrm{a}$ & $6.4 \mathrm{~b}$ & $1.6 \mathrm{a}$ & $16.2 \mathrm{a}$ \\
\hline
\end{tabular}

Nota: Prueba de Duncan al 95\%, para prueba de medias entre localidades

Pero los cambios en la dinámica poblacional de las plagas y enfermedades están mayormente asociados a las condiciones climáticas de la zona de estudio. Por ejemplo en Colombia, las plagas y enfermedades de los cítricos, se incrementan durante las épocas lluviosas [7]. En general, la mayor incidencia plagas y enfermedades, se presentó en la localidad de Manglaralto, comparado con Colonche. Sin embargo, los valores promedios solamente fueron estadísticamente diferentes en el caso de las hormigas (Cuadro 2). La presencia de estas plagas y enfermedades, no hace más que corroborar la importancia económica de éstas y la necesidad de diseñar adecuadas medidas de control, caso contrario podrían afectar negativamente a la cantidad y calidad de la cosecha. Bermejo [1], señala que en España, cuarto productor de cítricos a nivel mundial, uno de los principales factores que tienden a disminuir el rendimiento de la explotación, es el conjunto de plagas que atacan al cultivo.

\section{B.- Calidad del fruto del limón}

Los productores de limón asocian la calidad del fruto generalmente con el tamaño [8], es decir el diámetro y el peso. En Colonche, el diámetro mayor se reportó en el mes de setiembre, aunque la diferencia con los otros meses de evaluación, no 
fue muy grande, ya que el rango estuvo entre 3.5 a 3.7; mientras que en Manglaralto, el mayor diámetro correspondió al mes de noviembre, con un rango más amplio de valores que estuvo entre 2.5 a $3.2 \mathrm{~cm}$. En general el fruto tuvo un mayor diámetro en Colonche (3.58), con respecto a Manglaralto $(2.9 \mathrm{~cm})$, diferencia que fue estadísticamente significativa (Cuadro 3). Los valores encontrados en Colonche son más parecidos a los reportados en otros estudios en el Ecuador, como el de Caiza [3], que encontró valores entre 3.63 y $3.86 \mathrm{~cm}$ en estudio realizado en Manabí. Mientras que en México, para limón criollo se reportó diámetros de 3.66 a $3.72 \mathrm{~cm}$, Pérez, [9]. En la localidad de Colonche, el mayor peso se reportó en el mes de setiembre (45.7 g), la diferencia con los otros meses de evaluación fue notoria, ya que el rango estuvo entre 37.5 a 45.7; mientras que en Manglaralto, el mayor diámetro correspondió al mes de noviembre $(38.3 \mathrm{~g})$, con un rango poco amplio de valores que estuvo entre 36.9 a $38.3 \mathrm{~cm}$.

Cuadro 3. Diámetro del fruto de limón (cm) en Colonche y Manglaralto, provincia de Santa Elena, Ecuador.

\begin{tabular}{|l|l|c|}
\hline Zonas Agroecológica & \multicolumn{1}{|c|}{ Meses } & Promedio/mes \\
\hline \multirow{4}{*}{ Colonche } & Agosto & 3.6 \\
\cline { 2 - 3 } & Septiembre & 3.7 \\
\cline { 2 - 3 } & Octubre & 3.5 \\
\cline { 2 - 3 } & Noviembre & 3.5 \\
\cline { 2 - 3 } & Diciembre & 3.6 \\
\hline \multirow{4}{*}{ Manglaralto } & Agosto & 2.9 \\
\cline { 2 - 3 } & Septiembre & 2.9 \\
\cline { 2 - 3 } & Octubre & 2.9 \\
\cline { 2 - 3 } & Noviembre & 3.2 \\
\cline { 2 - 3 } & Diciembre & 2.5 \\
\hline Promedio Colonche & & $3.58 \mathrm{a}$ \\
\hline Promedio Manglaralto & \multicolumn{2}{|l}{$2.88 \mathrm{~b}$} \\
\hline \multirow{2}{*}{ Nota: Prueba de Duncan al 95\%, para prueba de medias entre localidades }
\end{tabular}

Cuadro 4. Peso del fruto de limón (g/unidad) en Colonche y Manglaralto, provincia de Santa Elena, Ecuador.

\begin{tabular}{|l|l|c|}
\hline Zonas Agroecológica & Meses & Promedio/mes \\
\hline \multirow{4}{*}{ Colonche } & Agosto & 40.4 \\
\cline { 2 - 3 } & Septiembre & 45.7 \\
\cline { 2 - 3 } & Octubre & 39.0 \\
\cline { 2 - 3 } & Noviembre & 37.5 \\
\hline \multirow{5}{*}{ Manglaralto } & Agosto & 37.3 \\
\cline { 2 - 3 } & Septiembre & 36.9 \\
\cline { 2 - 3 } & Octubre & 35.8 \\
\cline { 2 - 3 } & Noviembre & 38.3 \\
\hline Promedio Colonche & & $40.65 \mathrm{a}$ \\
\hline Promedio Manglaralto & & $37.07 \mathrm{a}$ \\
\hline
\end{tabular}

Nota: Prueba de Duncan al 95\%, para prueba de medias entre localidades

En general el fruto tuvo un peso mayor en Colonche (40.65), con respecto a Manglaralto (37.07), pero esta diferencia no fue estadísticamente significativa (Cuadro 4). Los valores encontrados en este estudio son parecidos a los reportados en otros estudios en el Ecuador, 
como el de Cevallos y Cevallos [2], que encontró valores entre 38.17 y $40.69 \mathrm{~g}$ en estudio realizado en Manabí. Mientras que en México, también para limón criollo se reportó pesos de 32.2 a $34.8 \mathrm{~g}$ [9].

\section{C.- Rendimiento del cultivo}

En la localidad de Colonche, el mayor peso/parcela se reportó en el mes de octubre (437 $\mathrm{kg}$ ), la diferencia con los otros meses de evaluación fue notoria, ya que el rango estuvo entre 105 a 437. En Manglaralto, el mayor peso/parcela correspondió también al mes de octubre $(262 \mathrm{~kg})$, con un rango de menor amplitud de valores ya que estuvo entre 105 a 262 . En general el rendimiento fue mayor en Colonche (5.42 t/ha), con respecto a Manglaralto (4.72 t/ha), pero esta diferencia no fue estadísticamente significativa (Cuadro 5). Los valores alcanzados en las dos zonas agroecológicas son bajos si los comparamos con otros reportes, como en México, donde el rendimiento va desde 14.6 a $26.6 \mathrm{t} / \mathrm{ha}$ [10]; mientras que en Piura (Perú) va desde 9 a 14 t/ha [12]. Los rendimientos por planta, también son bajos, tanto en Colonche $(21.7 \mathrm{~kg})$ como en Manglaralto $(18.9 \mathrm{~kg})$, si lo comparamos con los $73.8 \mathrm{~kg}$ reportado por Solís y Tomalá [11] también en Manglaralto.

Cuadro 5. Rendimiento en cultivo de limón en Colonche y Manglaralto, provincia de Santa Elena. Ecuador

\begin{tabular}{|l|c|c|c|c|c|c|c|c|c|}
\hline \multirow{2}{*}{$\begin{array}{c}\text { Zona } \\
\text { Agroecológica }\end{array}$} & \multicolumn{4}{|c|}{ Mensual (kg/parcela)* } & \multicolumn{4}{c|}{ Rendimiento Total } \\
\cline { 2 - 11 } & Ago. & Sep. & Oct. & Nov. & Dic. & Kg/planta & Kg/parcela & Kg/ha** & t/ha \\
\hline Colonche & 105 & 175 & 437 & 192 & 175 & 21.7 & 1084 a & 5420 & 5.42 \\
\hline Manglaralto & 227 & 175 & 262 & 175 & 105 & 18.9 & $944 \mathrm{a}$ & 4720 & 4.72 \\
\hline
\end{tabular}

Nota: Prueba de Duncan al 95\%, para prueba de medias entre localidades.

(*) En cada parcela se evaluó 50 plantas.

(*) Estimado con una densidad de $250 \mathrm{pl} / \mathrm{ha}$

La comparación de rendimiento entre países, son referenciales ya que en este estudio solamente se trabaja con la cosecha de cinco meses y algunos productores consideran que el limón en Ecuador se cosecha todo el año. Pero esto no está claro, ya que para autores como Lluiquinga [8], la cosecha a lo largo del año se debe a que las plantaciones tienen parcelas con diferentes estadios de desarrollo. Lo que si queda claro es la diferencia entre localidades, en el futuro será recomendable desarrollar proyectos para mejorar el manejo del cultivo en ambas localidades; pero de manera especial en Manglaralto.

\section{Conclusiones}

○ La mayor incidencia de plagas y enfermedades se presentó en la localidad de Manglaralto.

- El mayor diámetro del fruto, correspondió a la localidad de Colonche; diferencia que fue estadísticamente significativa con respecto a Manglaralto.

- El mayor peso del fruto, correspondió a la localidad de Colonche; pero la diferencia no fue estadísticamente significativa con respecto a Manglaralto.

- El mayor rendimiento, correspondió a la localidad de Colonche; pero la diferencia no fue estadísticamente significativa con Manglaralto.

\section{Recomendaciones}

- Desarrollar proyectos de mejora tecnológica en el cultivo de limón en la provincia de Santa Elena. Considerar aspectos diversos como fenología del cultivo, densidad de plantación, fertilización control de plagas y enfermedades, entre otros. 


\section{Bibliografía}

[1] Bermejo, J. 2011. Auto diagnóstico de plagas. Base de datos con imágenes y fechas actualizadas de las plagas agrícolas de España. Agrologica. Disponible

en: http://www.agrologica.es/cultivo/plaga-limonerolimon-citricos/

[2] Cevallos, D. y Cevallos, M. 2007. Evaluación de tres dosis de ácido giberélico sobre la calidad de limón criollo (Citrus aurantifolia L.) Swing. En pre y post-cosecha. En el sitio Bígua del cantón Jama, Manabí. Tesis Ing. Agr. Universidad Técnica de Manabí, EC.

[3] Caiza, J.; 1992. Estudio de fuentes y dosis de nitrógeno para el cultivo de limón criollo (Citrus aurantifolia L.) (Christm) Swingle, en la Provincia de Manabí. Tesis Ing. Agro. Portoviejo, EC. Universidad Técnica de Manabí. p. 40

[4] Falconí, C. 1999. Fitopatógenos. Enfermedades, plagas, malezas y nemátodos fitopatógenos de cultivos en el Ecuador. Centro de Diagnóstico y Control Biológico. Universidad San Francisco de Quito. 123 pp.

[5] Instituto Nacional Autónomo de Investigaciones Agropecuarias - INIAP. 2002. Estación Experimental Portoviejo. Sección Entomología. Informe técnico anual. Disponible en:http://repositorio.iniap.gob.ec/bitstream/41000/ 1215/1/INFORME\%20ANUAL\%20ENTOMOL OGIA\%202002.pdf.

[6] Instituto Nacional de Meteorología e Hidrología - INAMHI. 2014. Cambio Climático. Detección de cambio climático en el Ecuador. Disponible en: http://www.inamhi.gob.ec/ index.php/ clima/ boletines/anual.
[7] Instituto Colombiano Agropecuario, 2012. Manejo fitosanitario del cultivo de cítricos (citrus), medidas para la temporada invernal.

[8] Llumillanqui, J. 2010. Estudio de factibilidad para la creación de una empresa dedicada a la producción de limón Tahití ubicado en la provincia de Pichincha, sector Valle de los Chillos Barrio Fajardo. Tesis Ing. Comercial. Universidad Politécnica Salesiana. Quito. Ecuador. 186 pp.

[9] Pérez, O. 2002. Evaluación de mejoradores del suelo en limón mexicano. Universidad Autónoma de Chapingo. Chapingo, Méx. Terra Latinoamericana. Vol. 20, No. 003. p 337-346

[10] SAGARPA, 2014. Dirección general adjunta de planeación estratégica, análisis sectorial y tecnologías de información. México: Panorama del limón. Disponible en: http://www.uv.mx/veracruz/uvca366agronegocios-sustentables/files/2013/12/CoronaMonografia-julio2015.pdf

[11] Solís, L; Tomalá, M. 2010. Efecto de NPK en la Producción de Citrus Aurantifolia Swingle V. Sutil en la Zona de Sinchal - Barcelona, Cantón Santa Elena- Ecuador.

[12] Vegas, U. y Narrea, M. 2011. Manejo integrado del cultivo de limón. Jornada de Capacitación”. Universidad Nacional Agraria La Molina - AGROBANCO. Lima. 43pp. 